\title{
Control system for trials on material ship model
}

\author{
Janusz Pomirski, Ph. D., \\ Andrzej Rak, M. Sc., \\ Witold Gierusz, Ph. D., Assoc. Prof. \\ Gdynia Maritime University
}
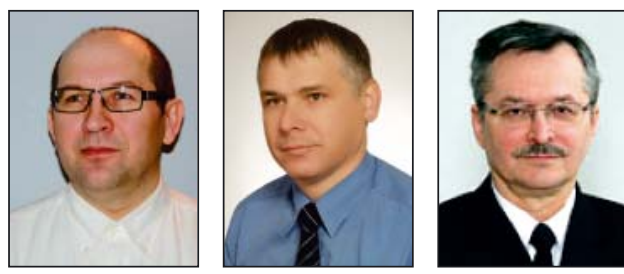
in-the-loop trials when a designed control system is tested inside a virtual environment instead of real actuators, disturbances, communication and measurement devices.

The paper presents software environement for fast prototyping and verification of motion control systems for ship. The environement is prepared for isomorphic reduced ship model which is used for training and in research in a area of ship motion control. The control system is build using Matlab-Simulink-xPC package which simplifies and accellerates design and verification of new control algorithms. The systems was prepared also for Hardware-

\section{ABSTRACT}

Keywords: ship controll Matlab; xPC Target; fast prototyping; hardware-in-the-loop; real-time control; computer simulation

\section{TRIALS ON A MATERIAL SHIP MODEL}

It is common rule that final verification of a designed control system should be performed in a real world on the real control object. Because of economical and safety reasons such trials of control systems for seagoing ships are difficult to perform. Unfortunatelly nowadays control systems engineers have to incorporate innovative technologies in short time which is limited by requirements of maritime industry. Therefore most of trials are made in computer simultion, when not all design factors can be tested and solved, so designed systems can not be fully robust and reliable.

Interesting option for verification of designed control systems are tests with reduced material model of ship. Using rules of mechanical similarities results obtained on the reduced material model can be scaled to those which can be obtained on an original ship. Ship Handling, Research and Training Center on the Silm Lake in Iława, Poland has many reduced ship models in scale 1:24 and 1:16 which are mainly used for a captains training. But since they have been equipped with additional control devices these models are perfect control objects for research purposes.

One of them called Blue Lady (Fig. 1) is used by research team from Department of Ship Automation at Gdynia Maritime Academy for verification of new control algorithms. Blue Lady is a reduced material model of the 176000 DWT VLCC (Very Large Crude Carrier) tanker in a scale 1:24 made of an epoxy laminate $[1,2,3]$. It has propulsion system powered by electric motors and fed from set of batteries. The model was made subject to the rights of geometric, kinematic and dynamic similarity, so the results and measurement data obtained on the lake can be scaled to the results recorded on a real tanker.

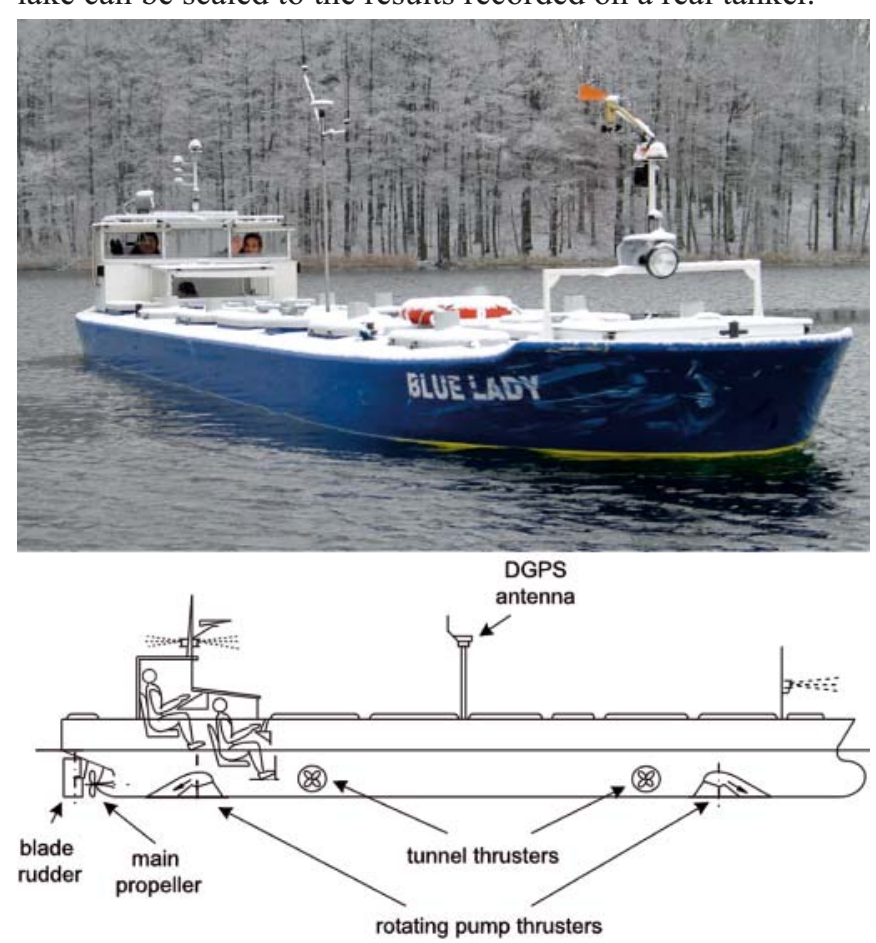

Fig. 1. VLCC "Blue Lady" tanker model as a training ship 


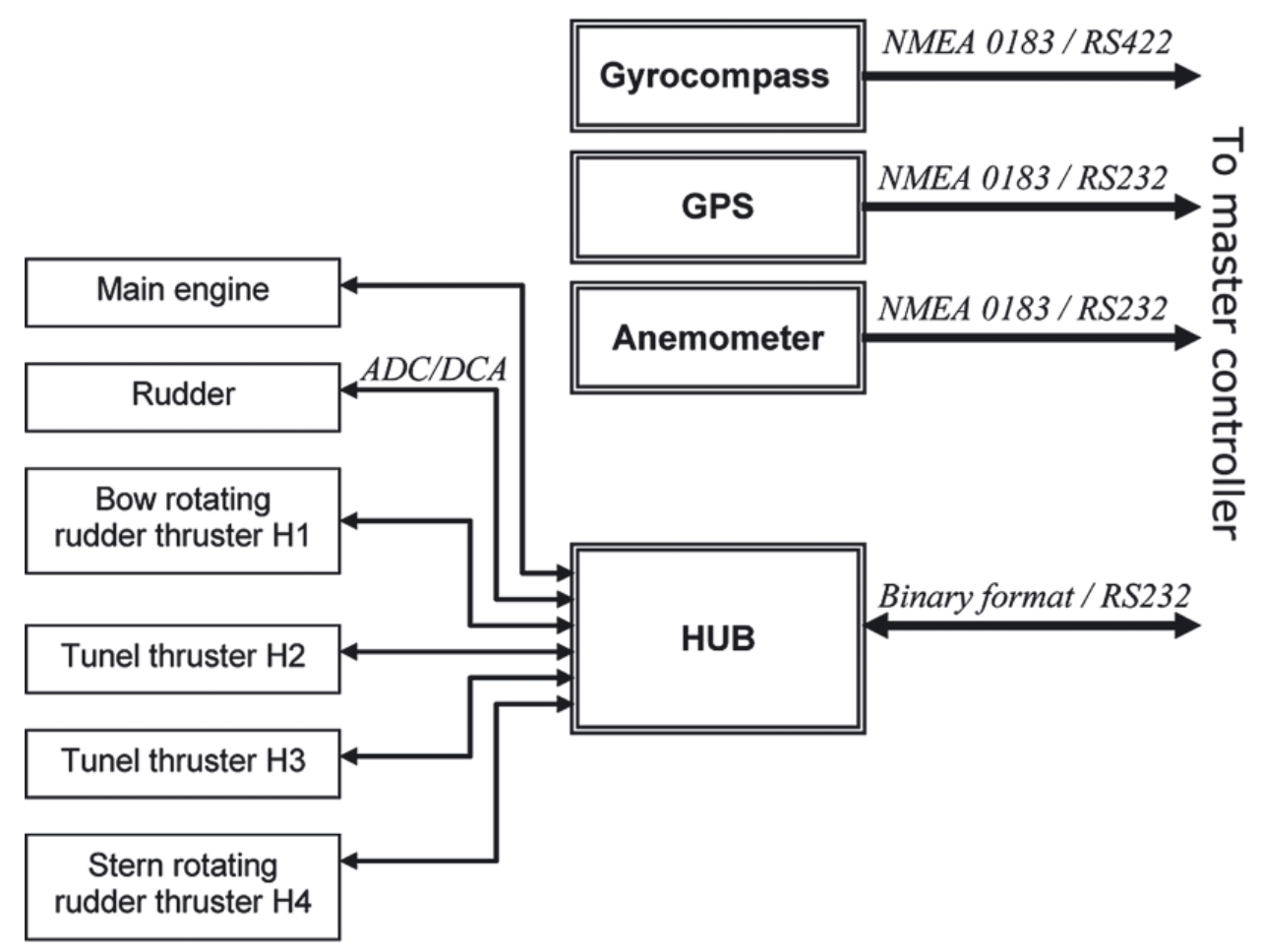

Fig. 2. Control equipment of the training ship Blue Lady

Fig. 2. presents devices used for automation control of the Blue Lady training ship: main engine with proppeler, conventional blade rudder and four variable speed jet thrusters: two of them are tunnel thrusters and other two are rotating thrusters with constrained turn angle. Direct control of actuators and measurement of real settings is performed by dedicated microcontroller system (called a hub) through set of 12-bit analog-digital and 10-bit digital-analog converters. The hub can cooperate with the master controller through bidirection interface RS-232. Data exchange format has form of binary records.

Moreover a gyrocompass for heading measurement, a ultrasonic anemometer for wind disturbances, and DGPS (Differential GPS) receiver with a reference station fixed on a lake shore - for recording of actual position are installed on the training ship. These devices transmittes data in NMEA0183 format through RS232/422 interfaces

\section{CONTROL SOFTWARE}

For scientist and engineers in a field of automation control Mathwork's MATLAB is well known and frequently used programming package. Matlab users can make calculations with its programming language with many toolboxes and also can simulate control systems behaviour with the powerful Simulink. Less known is knowledge that some toolboxes gives possibility to control systems in a real time. The toolboxes that are used for building of real control systems are Simulink Coder (former Real Time Workshop) [4] and xPC Target [5].

Simulink Coder creates ANSI/ISO C code from given Simulink diagram and Matlab functions. The code can be used for developing and testing purposes, particulary for acceleration of simulation, rapid protoyping, real-time applications and hardware-in-the-loop testing. User can tune and monitor the generated code using Simulink or run and interact with the code outside MATLAB and Simulink. The code created by Simulink Coder can be compiled to the executable for many target platforms.
Using xPC Target toolbox the code generated by Simulink Code can be executed on remote computer, which become a real time controller. xPC Target provides a real-time kernel and a library of drivers to include in a Simulink diagram. xPC Target supports a comprehensive selection of $\mathrm{I} / \mathrm{O}$ which includes: analog and digital inputs/outputs in many formats, serial communication ports (RS232/422/485), incremental encoder, Ethernet (UDP/IP, real-time raw Ethernet), video cards (USB WebCam, CameraLink), communication data buses and protocols (e.g. CAN) and more. The toolbox additional features are a host-target interface for real-time monitoring, parameter tuning, and data logging. xPC Target computer can work under constant monitoring of an master computer running Matlab software but also it is possible to generate a stand-alone software which boots PC and then works without any supervision.

Popularity of Matlab makes it optimal choose for preparation of a control setup for a research purposes. Transition from pure computer simulation to real application is much easier, because the same Simulink diagrams used in simulation are used in generation of control software for final controllers. Moreover since performance of nowadays microprocessors still fast increase, even developing of commercial, industry control systems with Simulink-xPC Target toolbox becomes interesting option, because time of obtaining of a final solution becomes the most important factor in a design process.

For building of xPC Target control systems two PC-type computers are required. One of them plays role of supervising computer (host) and runs MS Windows operation system and Matlab-Simulink software. Because of reqirements of nowadays Matlab version this PC have to be rather fast unit. Second PC computer (target) is used as a hardware platform which runs dedicated, propertiary real-time kernel and control software. Creator of Matlab - Mathworks presents xPC Target Turnkey programme which combines xPC Target with a high-performance real-time target machine and $\mathrm{I} / \mathrm{O}$ modules. Target machines and $\mathrm{I} / \mathrm{O}$ modules meet a wide range of $\mathrm{I} / \mathrm{O}$ connectivity, form factor, and environmental requirements. 
Also using of a standard low-cost computer is possible, but it is recomended to use a dedicated and suported by xPC Target toolbox extension cards, because writing of software drivers for non-standard extension cards requires much programmer experience. Target computer performance should be selected according to control algorithms requirements, but in many cases is not critical factor.

Both computers: host and target have to be equipped with an Ethernet networks adapters and connected together via TCP/IP protocol. The network junction is used for control soffware transfer from host to target computer, and also for monitoring and supervising. It is possible to generate stand-alone software for target. In this configuration target computer boots real-time kernel and loads control software directly from a hard disk.

Fig. 3 presents how Matlab can be used during design process of a new control algorithms. In a first stage of a design Simulink is powerful tool for off line data processing, identification of a mathematical model of a process and fast verification of designed controllers. In a last third stage the final verification is done during trials on a lake, where a PC controller is powered by a PC-Target real-time kernel which executes controll algorithm defined by a Simulink diagram. As a graphical Simulink diagram can be simply modified therefore such software can be called a real fast-prototyping tool.

Another speed-up is introduced by a middle second verification stage so called Hardware-in-the-Loop (HiL). Real control system consists not only of controler but also it consists of measurement systems, actuators, communication interfaces, etc. HiL is a methodology of testing a whole controller's functionality and communication. HiL significantly reduce development time of control systems by testing the controller inside a virtual environment instead of real actuators, disturbances, communication and measurement devices, so all hardware and software components of control system can be tested before final trials.

\section{RESEARCH SETUP}

As shown on figure 2 training ship Blue Lady requires four RS232/422 serial ports for communication with supervision controller. Therefore a four-port multi I/O PCI card has been installed in the xPC Target computer. The card was selected

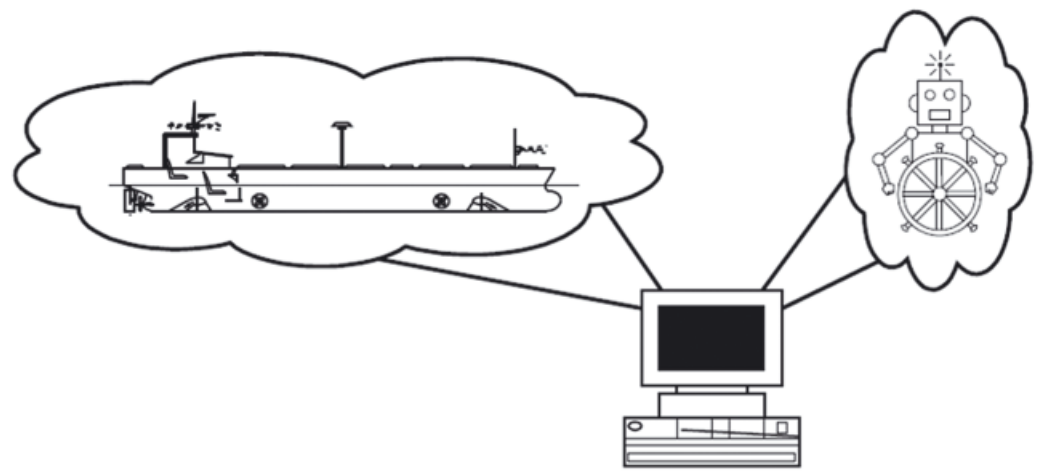

Stage I

Computer

simulation

Ship mathematical model and controller

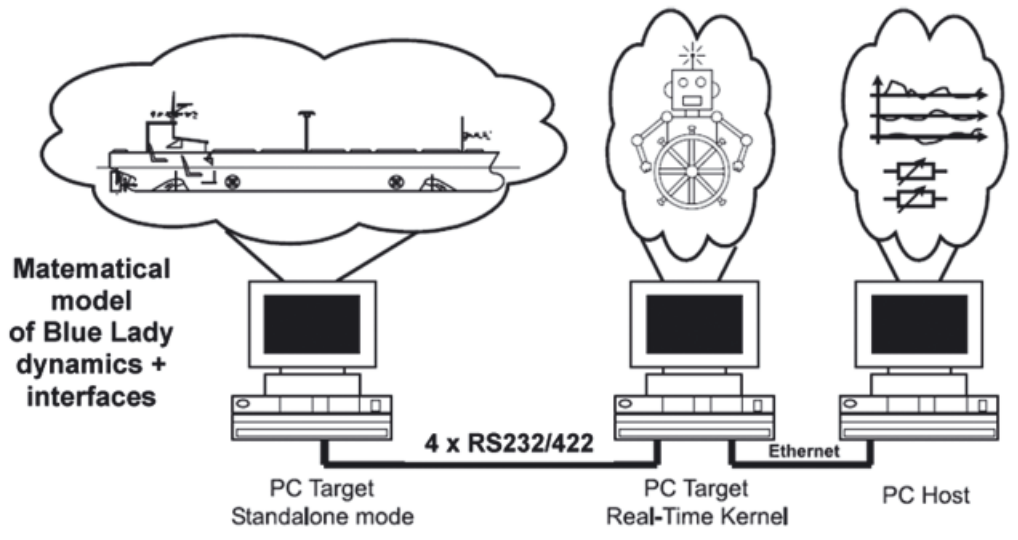

Stage II

Hardware-in-

the-loop trials

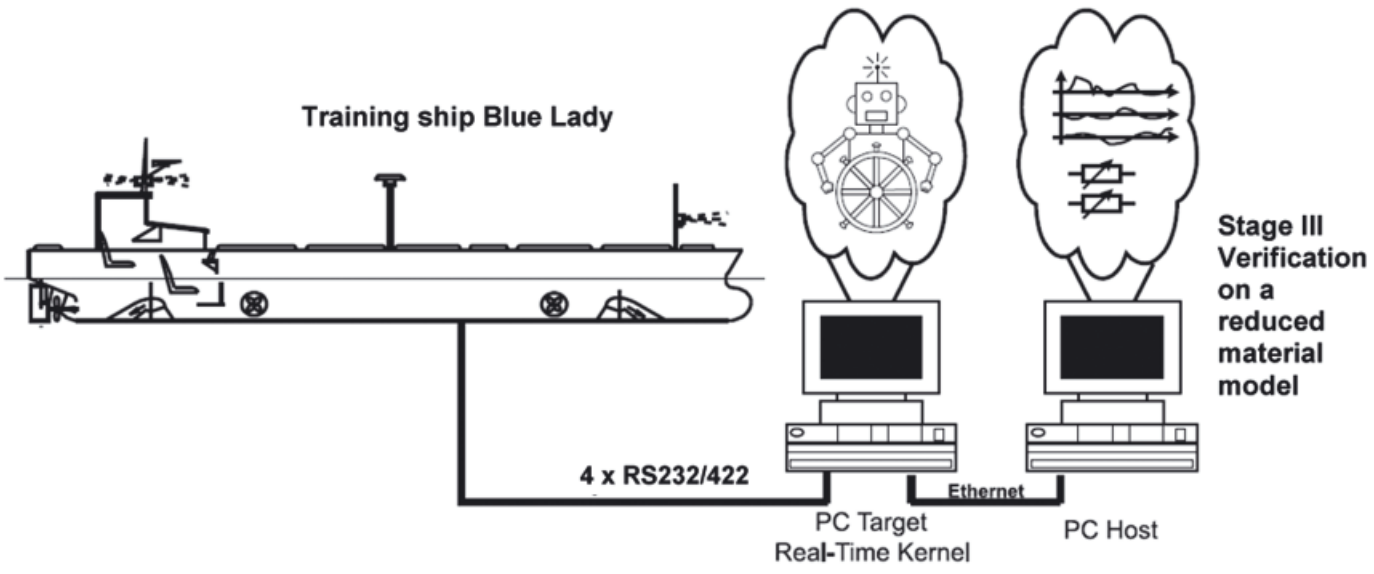

Fig. 3. Verification of control algorithms for ships 
from those recomended by Matlab - it is QSC-100-d9 four-port RS-232 Multiport Serial Adapter from Quatech. Converting between RS232 and RS422 interfaces is done by additional external converter.

The XPC serial port driver works with streams of bytes which represent data received from serial port or transmitted to a port. Data streams from receiver must be decoded according to a proper data format. Gyrocompass, GPS and anemometer send data in a NMEA 0183 ASCII format which can be decoded using standard 'ASCII Decode' block, where format string is defined in a similar way like in function sprintf in a $\mathrm{C}$ language.

Data received from the hub is packed into binary record which consists of:

- two-byte header 'BL';

- 14 word-size numbers from A/D converters which measure actual control parameters, valid are only 12 less significant bits in every number;

- 5 bytes from digital inputs;

- control byte which is sum modulo 256 of all previous bytes in the record.

The multi I/O card sends only only data to the hub in form of binary record which consists of:

- three-byte header 'MP ';

- 8 word-size numbers for D/A converters which controls actuators (valid are only 10 less significant bits in every number);
- 5 bytes for digital outputs;

- control byte which is sum modulo 256 of all previous bytes in the record.

For the purpose of trials on ship models universal template diagram in Simulink have been developed. The diagram is called a sceleton and is shown in Fig. 4.

The template diagram contains the drivers for Multi I/O with four RS232 ports and boxes which represents data decoders and encoder (Figs 5, 6, 7). All data from input decoders are brought to the box Control Algorithm, where Simulink diagram which represents a verified algorithm will be included. Each team member has the ability to insert and modify his/her algorithm which is currently working on: to control the course of the vessel, its speed, movement on the trajectory and dynamic positioning, etc. and no others part of the sceleton have to be modified. A simple PID course controller is shown as an example of control algorithm on figure 8. Output from the Control Algorithm is brought to Output Encoder, which pack data that are send to the hub and controls ship actuators.

The skeleton also provides monitoring of all decoder outputs, encoder inputs from target computer into a Matlab workspace in a host computer. This data can be processed by additional Matlab scripts and stored on your hard disk for later processing.

Target scopes are components which enables local monitoring facilities for selected parameters on a screen of target xPC computer.

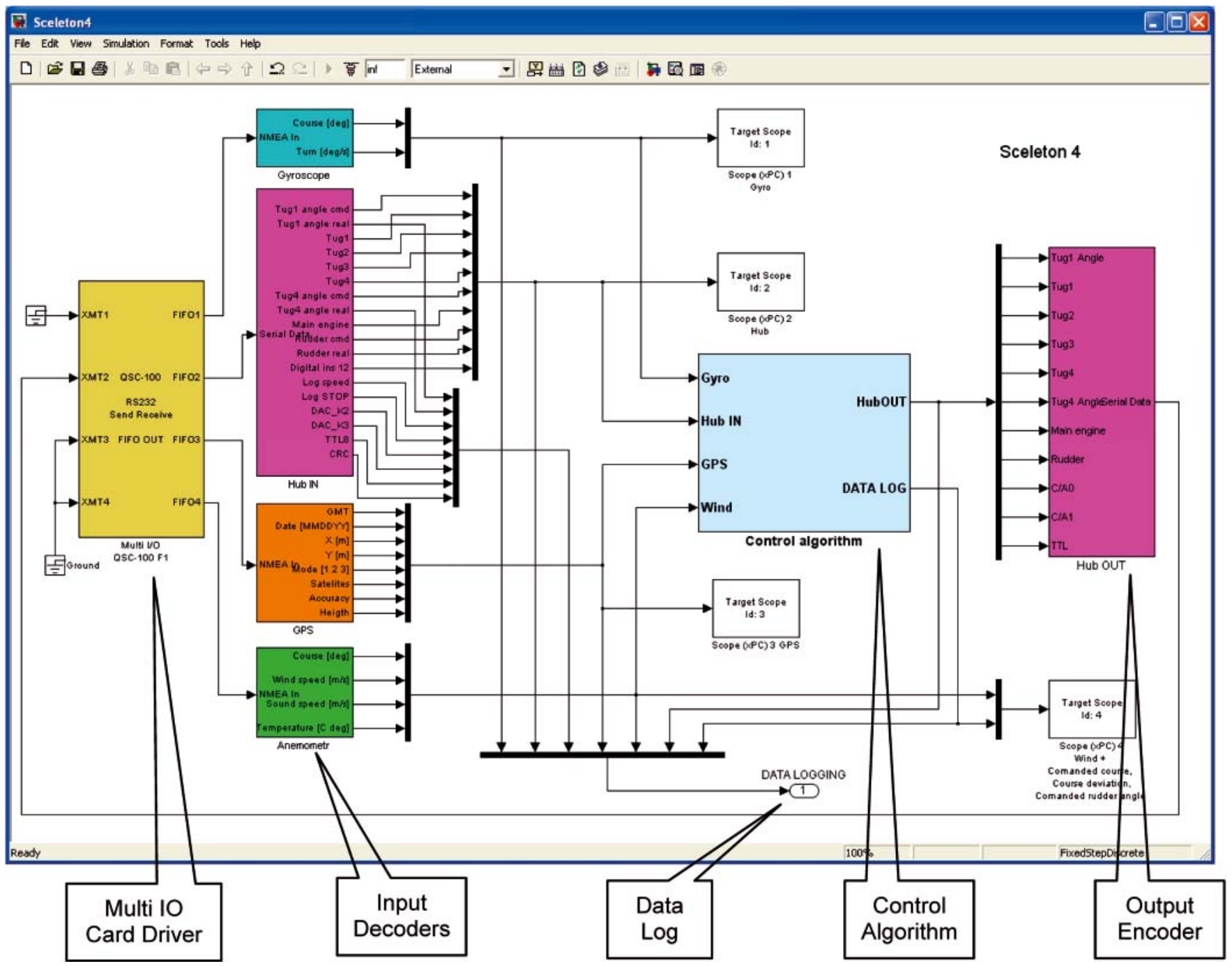

Fig. 4. Sceleton - Simulink control framework used in trials 


\section{HARDWARE-IN-THE-LOOP SIMULATION}

For Hardware-in-the-Loop (HiL) trials a virtual environement of system on the Silm lake has also been build using Matlab-Simulink-xPC Target tools. Devices and dynamics of a real traing ship are simulated in a separate PC. This PC also runs xPC Target software, but standalone configuration has be selected, because after design the model is rarely changed.

Simulink model of the training ship Blue Lady is based on the set of non-linear equations, which describes dynamics of actuators dynamics, forces and moments acting the ship, hull dynamics and kinematics (Fig. 9) [1, 2]. Simulink model is created in a form of C-language $\mathrm{S}$-function and also is used for computer of-line simulation of the first design stage (Fig. 3).

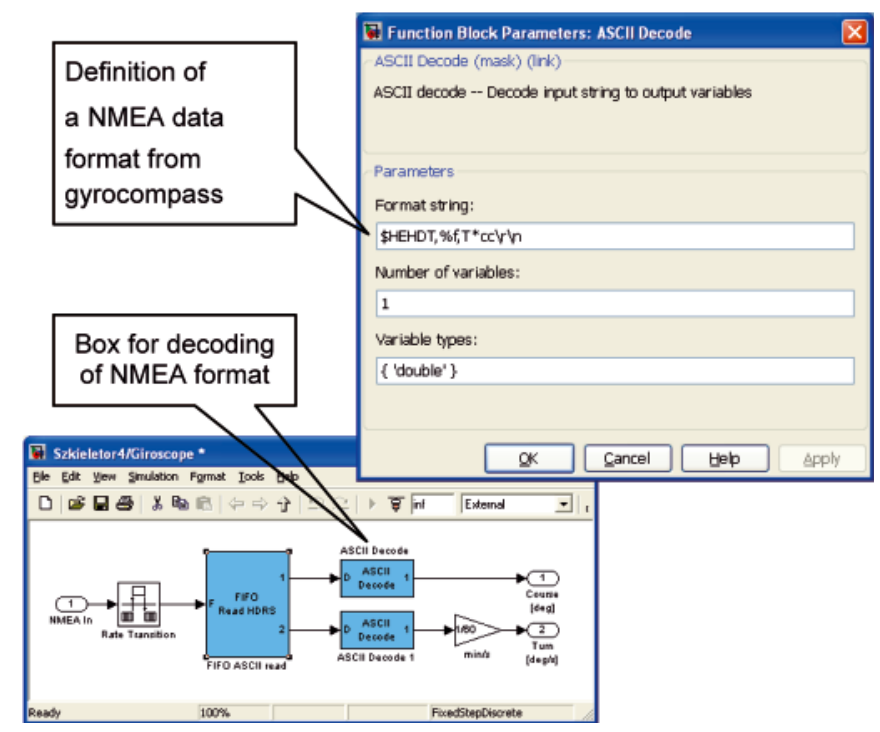

Fig. 5. Definition in Simulink of a decoder block of a NMEA 0183 format record from gyrcompass

Simulink diagram prepared for Hardware-in-the-Loop trials (fig.10) includes some elements which fit parameters units from the Blue Lady model to those required by training ship communication interfaces (Fig. 2). Decoder and encoder

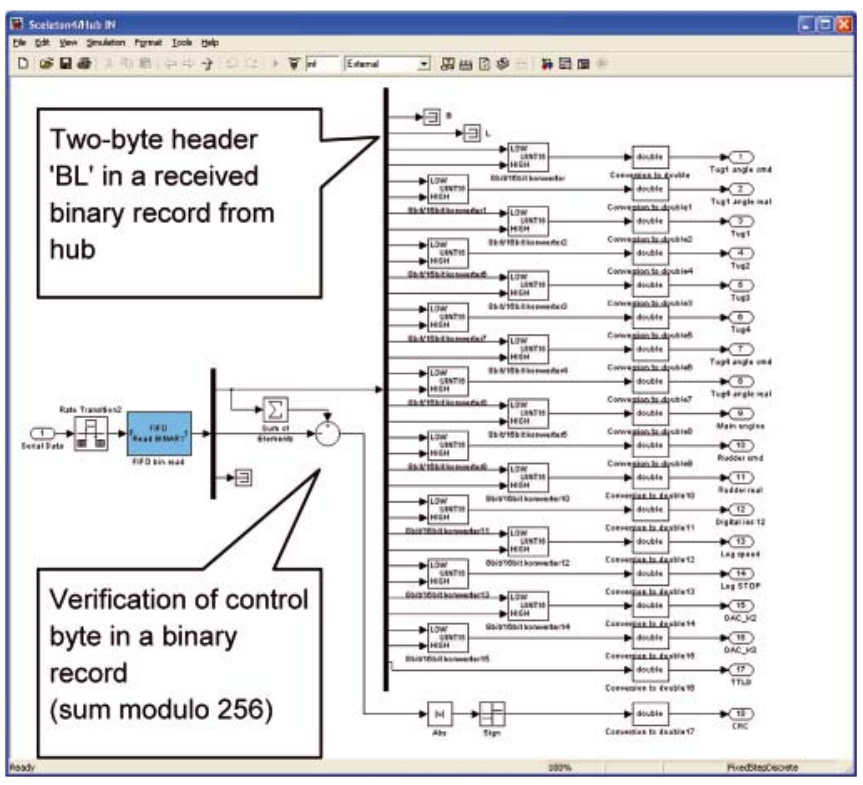

Fig. 6. Definition in Simulink of a decoder block of a binary format record received from hub blocks in HiL diagram are revers counterparts of blocks in the sceleton.

HiL stage drew up conditions for the simulation to the reality of the ship's control. Circuit connections between the system control and object control are identical with those of the "Blue Lady". In both cases the same Simulink block diagrams of the environment, the same compilers and starting procedure are used. Introduction of this stage saves a lot time for pretesting of systems, debuging diagrams, verifing communication interfaces.

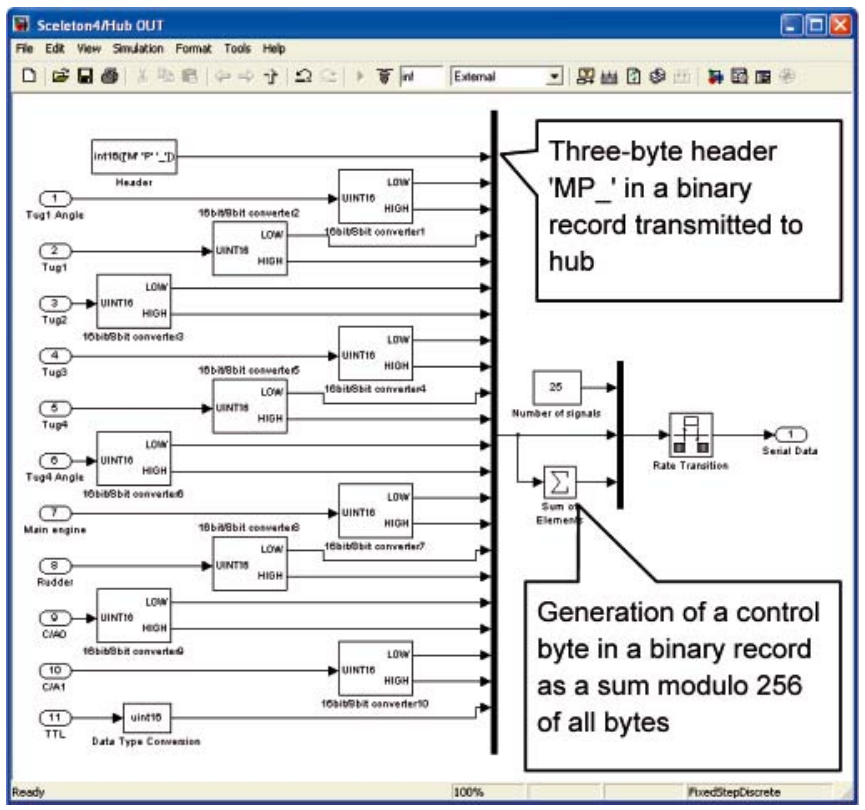

Fig. 7. Definition in Simulink of a encoder block for generation of binary format records transmitted to the hub

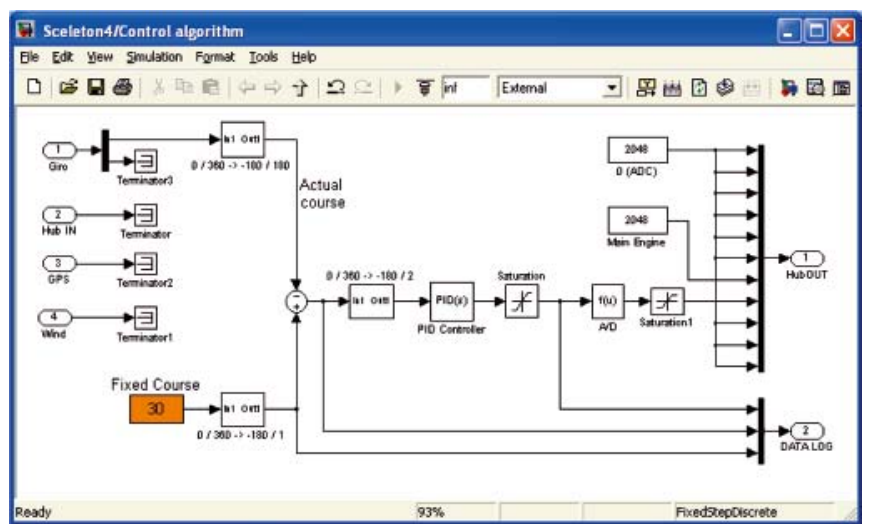

Fig. 8. An example of control algorithm in a scelleton PID heading controller

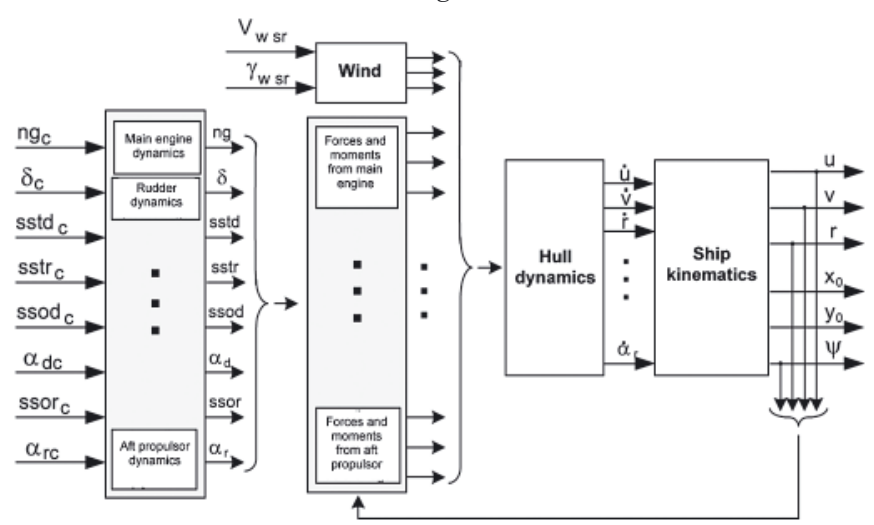

Fig. 9. Multidimensional Dynamics model of the training ship "Blue Lady" [1, 2] 


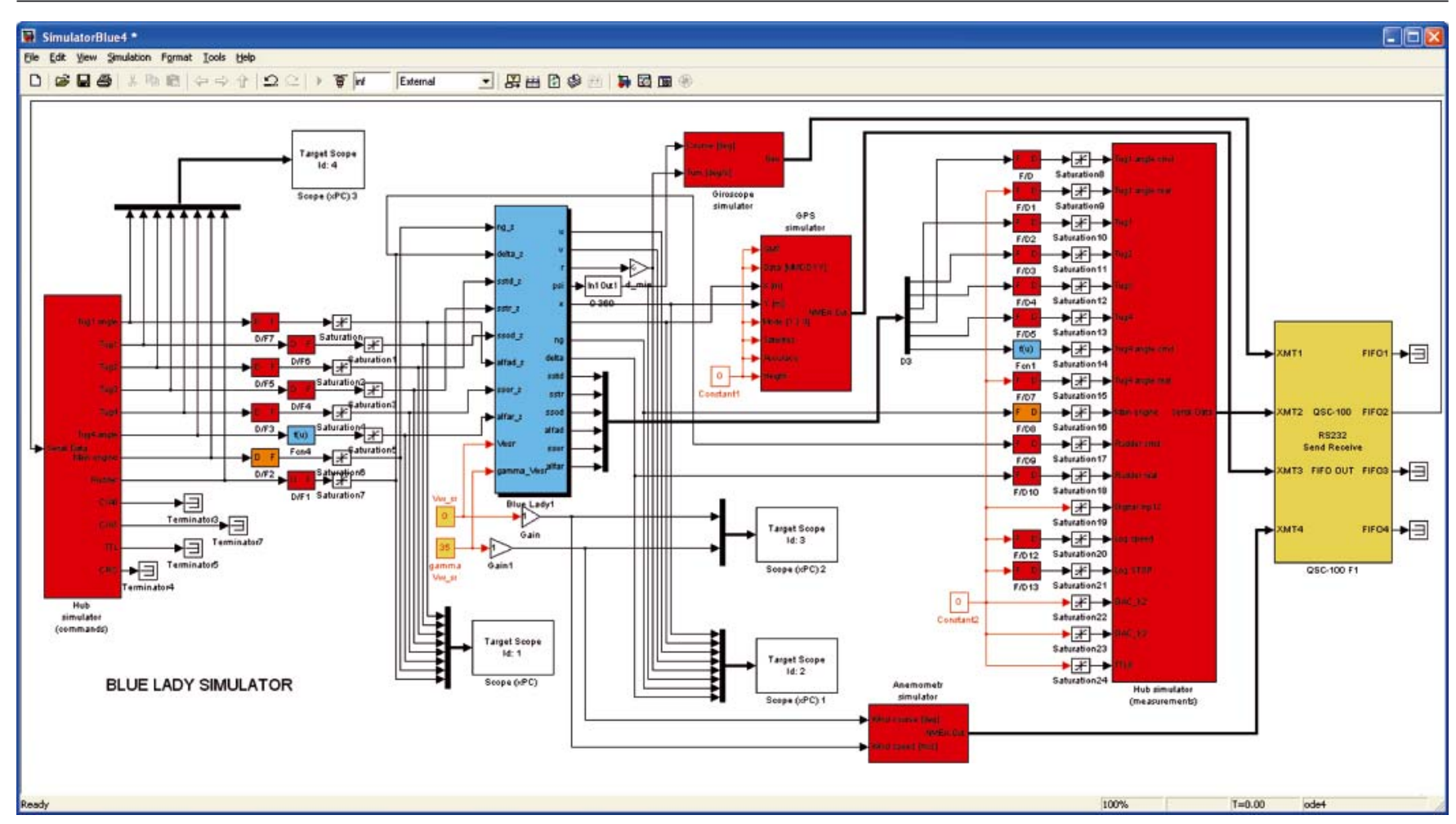

Fig. 10. Simulator diagram of Blue Lady training Ship used for target xPC for Hardware-in-the-Loop trials

\section{CONCLUSIONS}

Matlab with its toolboxes Simulink, Simulink Coder, xPC Tatget is powerful software package for building a useful design environement in the area of a real-time control so Matlab enables posibility for rapid prototyping of control systems of ship movements. It is possible to build a multistage research (Fig. 3) test bed where proposed control algorithms can be tested in a computer simulation then during hardware-in-loopsimulations and finally during trials in a real environement. Very interesting option is replacing tests on real ships with trials in a reduced scale environement, when real ships are replaced by the reduced matrial ship models. The models are build in such way that rules of mechanical similarities are preserved so results for the reduced scale and real environement can be compared.

\section{BIBLIOGRAPHY}

1. Gierusz W.: Synteza wielowymiarowych uktadów sterowania precyzyjnego ruchem statku $z$ wykorzystaniem wybranych metod projektowania ukladów odpornych, Gdynia, 2004.
2. Gierusz W.: Simulation model of shiphandling training boat "Blue Lady", Proc. Of the IFAC CAMS Conference, Glasgow, UK, 2001.

3. Morawski L., Pomirski J.: Identification and control of a direction unstable ship, Problems of Nonlinear Analysis in Engineering Systems, 1(13), vol.7, Kazan, 2001.

4. Simulink Coder. Users Guide. MathWorks, USA

5. xPC Target. Users Guide. MathWorks, USA

\section{CONTACT WITH THE AUTHOR}

Janusz Pomirski, Ph. D., Andrzej Rak, M. Sc.,

Witold Gierusz, Ph. D., Assoc. Prof. Faculty of Marine Electrical Engineering, Gdynia Maritime University, Morska 81-87

81-225 Gdynia, POLAND e-mail: jpomir@am.gdynia.pl e-mail: anrak@am.gdynia.pl e-mail:wgierusz@am.gdynia.pl 